(2) Open Access Full Text Article

\title{
Sensorimotor polyneuropathy and foot-drop as result of a prostate cancer paraneoplastic syndrome
}

This article was published in the following Dove Press journal:

Research and Reports in Urology

4 December 2013

Number of times this article has been viewed

\author{
John J Bodkin' \\ Michael Duff ${ }^{1,2}$ \\ Phillip J Seereiter J ${ }^{3}$ \\ K Kent Chevli 1,3
}

'State University of New York School of Medicine and Biomedical Sciences Department of Urology, Buffalo;

${ }^{2}$ Cancer Care of Western New York, Cheektowaga; ${ }^{3}$ Western New York Urology Associates, Cheektowaga, NY, USA
Correspondence: Michael Duff

Cancer Care of Western New York, 3085 Harlem Rd, Cheektowaga, NY 14225, USA

Tel + I 7168445500

Email mduff@cancercarewny.com
Abstract: Paraneoplastic syndromes (PNS) vary in incidence and manifestation based on tumor histology. PNS secondary to urologic malignancies have an extremely low incidence. Most reported cases of PNS from urologic malignancies are associated with adenocarcinoma. Peripheral neuropathy-associated PNS from urologic malignancy are exceedingly rare. An 80-year-old male developed a paraneoplastic sensorimotor polyneuropathy and foot-drop after a diagnosis of clinical stage T2 2 N0M0, Gleason grade $5+4$ prostate cancer. A thorough workup is needed in order to adequately assess and treat PNS. Careful analysis must be used to determine the root cause of a patient's symptoms.

Keywords: prostate cancer, paraneoplastic syndrome, intensity-modulated radiation therapy, peripheral neuropathy

\section{Introduction}

Paraneoplastic syndromes (PNS) are defined as a collection of symptoms and clinical signs occurring in cancer patients involving systemic effects taking place remotely from the tumor. They are not attributable to direct compression or invasion by the tumor or to adverse effects from infection, nutritional deficits, or treatment. ${ }^{1,2}$ PNS are classified according to their clinical manifestations (ie, endocrine, hematologic, neurologic, dermatologic, inflammatory). ${ }^{1}$ Neurologic PNS occur in $6 \%$ of all patients with cancer. ${ }^{2}$ PNS are seldom observed with prostate cancer. ${ }^{3}$ Only a few cases have been reported in the literature. Here we describe the case of a man with adenocarcinoma of the prostate who developed a sensorimotor polyneuropathy and foot-drop as a result, and review the literature.

\section{Case report}

An 80-year-old male with a history of gastroesophageal reflux disease, benign prostatic hyperplasia, and diabetes presented with 2 days of gross hematuria, worsening hesitancy, and difficulty urinating. Prior to this, the patient had had a 6-month history of lower urinary tract symptoms for which his primary medical doctor had prescribed an $\alpha 1$ receptor antagonist at a dose of $0.4 \mathrm{mg}$ orally once daily. This initially brought about brief mild improvement in the patient's symptoms, but ultimately there was an increase in lower urinary tract symptoms. The patient denied renal colic, flank pain, or any constitutional symptoms. He had not suffered any recurrent urinary tract infections in the past and denied taking any anticoagulants other than two aspirin occasionally for headache. 
On presentation to the urology clinic, the patient was found to have severe obstructive urinary symptoms. Rectal examination revealed a grossly abnormal prostate gland with nodularity throughout the entire palpable surface. Laboratory information showed a prostate serum antigen of $20.2 \mathrm{ng} / \mathrm{mL}$. His most recently documented prostate serum antigen was $8.5 \mathrm{ng} / \mathrm{mL}$, which was from one year previously. Baseline blood urea nitrogen/creatinine was $32 / 0.87$. Because the patient had obstructive symptoms and a post void residual of $319 \mathrm{~mL}$, a Foley catheter was placed and he was scheduled for a cystoscopy (as part of the hematuria workup)/voiding trial. He was also put on silodosin $8 \mathrm{mg}$ per day orally and ciprofloxacin $500 \mathrm{mg}$ orally twice daily for 7 days. Upon the patient's return to the office, his Foley catheter was draining adequately. Post void residual was $600 \mathrm{~mL}$. Cystoscopy showed a large amount of clot in the base of the bladder, which was irrigated to clear, and no active bleeding was noted. Significant distortion and posterior impingement of the trigone was noted, left hemitrigone being greater than the right. No diverticulum or stones were noted. Prostatic urethra showed a near visually obstructing prostate but no intraluminal filling defects of the bladder. A computed tomography (CT) urogram showed left hydronephrosis down to the trigone and some mild nonspecific adenopathy. Urine culture and sensitivity was positive for a Proteus urinary tract infection. The patient was already on ciprofloxacin (which was shown to be sensitive) and responding to treatment. Bicalutamide $50 \mathrm{mg}$ orally once daily and finasteride $5 \mathrm{mg}$ orally once daily were prescribed.

The patient underwent transrectal ultrasound and sampling of the prostate gland. It was noted that the patient's prostatic volume was $14.6 \mathrm{cc}$. Due to the patient's age, gross hematuria, and comorbid conditions, a limited six-core prostate biopsy was performed. Biopsy showed six of six cores positive for an adenocarcinoma Gleason grade $9(5+4)$ prostate cancer (see Figure 1). CT scan and bone scan did not show any evidence of metastatic spread. The patient was diagnosed with clinical stage $\mathrm{T} 2 \mathrm{cN} 0 \mathrm{M} 0$ prostate cancer. The patient completed and passed a voiding trial. Bicalutamide, silodosin, and finasteride were continued. A depot suspension of leuprolide acetate (30 mg intramuscularly every four months) was started. Calcium and vitamin D were added for additional support (entirely prophylactic, given that the patient had no vitamin deficits prior to starting leuprolide). At this stage in the patient's clinical course, his urinalysis was normal and his post void residual was down to $67 \mathrm{~mL}$. The

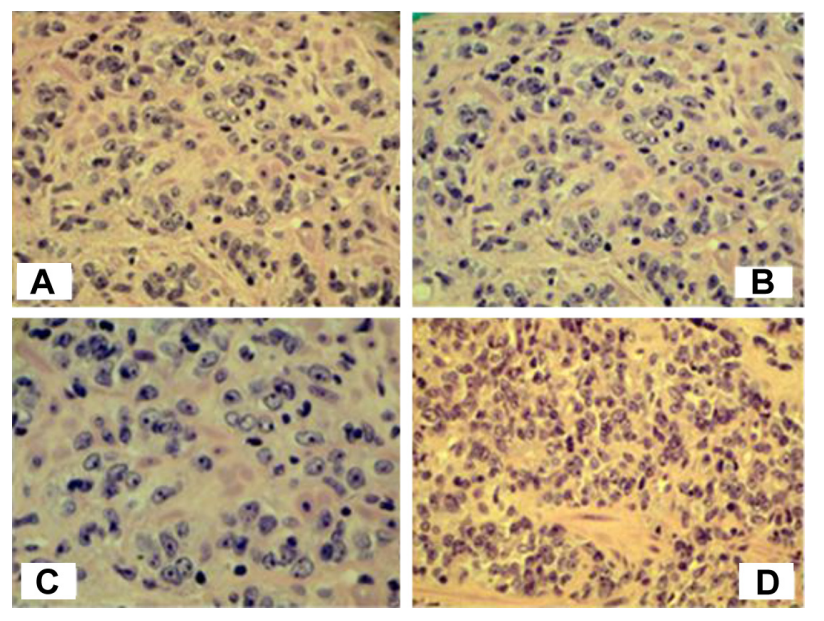

Figure I Prostate biopsy - Gleason $9(5+4)$ prostate cancer. (A) Hematoxylin

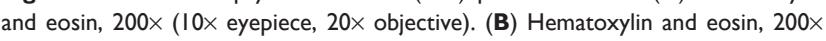
(I0X eyepiece, $20 \times$ objective). (C) Hematoxylin and eosin, $400 \times$ (I0× eyepiece, $40 \times$ objective). (D) Hematoxylin and eosin, $200 \times($ (10× eyepiece, $200 \times$ objective).

patient was counseled on the management options and chose intensity modulated radiation therapy (IMRT) as treatment for his prostate cancer.

Fiduciary gold seed markers were placed. The patient underwent a planning CT scan which was fused with a magnetic resonance imaging (MRI) scan. The prostate and seminal vesicles were contoured with a planning CT scan, expanded into planning target volumes, and a radiation plan was developed. The patient received 46 treatments over 64 days for a total dose of 8,280 cGy. Phase 1 (planning target volume 1) consisted of the prostate, seminal vesicles, and regional lymph nodes receiving 4,500 cGy in $180 \mathrm{cGy}$ daily fractions. Phase 2 (planning target volume 2) consisted of the prostate alone receiving 3,780 cGy in $180 \mathrm{cGy}$ daily doses. Intensity-modulated radiation therapy using an image-guided linear accelerator (Varian Instruments Inc, Palo Alto, CA, USA) was utilized to minimize both acute side effects and long-term sequelae. The patient tolerated his treatments well. Mild urinary symptoms were experienced, but his urination had been improving and his international prostate symptom score was $<15$ (9). Therefore, the patient did not require any additional medications and we felt that any surgical intervention (such as transurethral resection of the prostate) for symptomatic relief was not warranted at this time. The patient did develop some bowel symptoms that required a change in diet.

The patient was examined by his urologist post radiation therapy and was clinically doing well. He had a post void residual of $104 \mathrm{~mL}$ and a significantly reduced prostate serum antigen of $0.4 \mathrm{ng} / \mathrm{mL}$. Repeat renal and bladder 
ultrasound showed resolution of his previously noted left hydronephrosis. However, just after intensity-modulated radiation therapy ended, the patient began to experience right lower extremity weakness and intermittent numbness from the mid calf distally. The patient had not experienced any falls or trauma. His serum glucose was under control throughout his entire evaluation (prior to treatment it was noted to be $122 \mathrm{mg} / \mathrm{dL}$ and then post treatment measured $88 \mathrm{mg} / \mathrm{dL}$ ). This progressed to require an evaluation by a neurologist. Physical examination showed a mild widebased stance with decreased stride length and a tendency to circumduct the right lower extremity, with mild to moderate right foot-drop. Initial evaluation included a lumbar puncture, comprehensive metabolic panel, complete blood count, urinalysis, positron emission tomography scan, renal ultrasound, and CT scans of the head and spine. All initial studies were negative. Further evaluation included an electromyogram, as well as an MRI scan of the brain/ cervical and thoracic spines. MRI of the brain specifically showed only mild diffuse cortical atrophy. The cervical and thoracic spine MRI scans showed a T2 hyperintensity spanning the lateral cortical spinal tracts of the entire cervical and thoracic cords.

Blood work was collected locally, sent to the Mayo Clinic, and profiled in their paraneoplastic autoantibody evaluation. The profile was consistent with organ-specific neurologic autoimmunity, which suggested an association with multifocal neurologic mainfestations. ${ }^{4}$ Specifically, the blood work revealed a positive neuronal voltage-gated potassium channel antibody. These findings combined with MRI of the spine led the neurologist to diagnose the patient with a paraneoplastic syndrome. Magnetic resonance neurography was not done. The patient's nerve pain was based solely on his personal perspective. The patient was given an ankle-foot orthosis and a cane for ambulation, was started on intravenous immunoglobulin therapy, and referred for home-based physical therapy. At this time, the patient felt that both his strength and ambulation had improved, and his physical therapist agreed. The patient has not experienced any new complaints.

\section{Discussion}

A paraneoplastic phenomenon usually arises from: biologically active substances (hormones, hormone precursors, or hormone-like substances) aberrantly produced by the underlying neoplasm; modulation of the immune system via autoimmunity, production of immune complexes, and immune suppression; and unknown causes. ${ }^{2}$ Paraneoplastic syndromes are classified according to their manifestations. Neurologic PNS occur in the lowest incidence of any of the manifestations. Neurologic PNS can lead to severe neurologic decline, physical debilitation, and even death. The tumor in this patient's case was an adenocarcinoma, which most likely expressed neuronal antigens that are recognized as "non-self" by the dendritic cells of the body. The generation of neuronal antibodies to these antigens by plasma cells results in immune-mediated damage that can affect peripheral neurons leading to a sensorimotor peripheral neuropathy. ${ }^{5}$ A detailed literature search reveals very few cases involving a neurologic PNS resulting from a prostate cancer ever being reported.

Much of the literature reviews report PNS occurring before, and thus leading to a diagnosis of cancer. Paraneoplastic manifestations may be mistaken for metastatic disease, leading to inappropriately aggressive therapy. Conversely, symptoms of true metastasis may be attributed to PNS, thus delaying therapy. ${ }^{6}$ However, our patient's symptoms manifested shortly after treatment for his prostate cancer was complete. He was given a thorough workup so as to not misinterpret the clinical signs. His particular abnormal findings and the lack of any other abnormalities on workup proved to be consistent with a paraneoplastic myelopathy, resulting in the patient suffering a right lower extremity weakness and foot-drop.

Treatment of a PNS is entirely on a case-by-case basis. Most treatment plans begin with aggressive treatment of the underlying cancer. In this patient's case, that was already achieved. The patient was given treatment with intravenous immunoglobulin starting at $2 \mathrm{~g} / \mathrm{kg}$ infused over 5 days, followed by $0.6 \mathrm{~g} / \mathrm{kg}$ every 4 weeks for 2 months and home physical therapy. At 2-month follow-up, the patient showed some resolution of weakness as well as improved ambulation. The fact that this patient had the outcome of the workup that he did, and his right lower extremity weakness was responding to intravenous immunoglobulin and physical therapy strongly mitigate against metastatic disease. Further, detection of voltage-gated potassium channel antibodies against antineuronal antigens via the Mayo Clinic PNS assay was strongly suggestive of a neurologic PNS.

\section{Disclosure}

This case report was reviewed and written without any funding. The authors have no conflict of interest to disclose in relation to this work. 


\section{References}

1. Gakiya M, Naka H, Saito S. Paraneoplastic neurological and hematological syndromes associated with prostate cancer. Int J Urol. 2012;19:471-474.

2. Sacco E, Pinto F, Sasso F, et al. Paraneoplastic syndromes in patients with urological malignancies. Urol Int. 2009;83:1-11.

3. Berger JR, Bensalem M, Dalmau J. A brainstem paraneoplastic syndrome associated with prostate cancer. J Neurol Neurosurg Psychiatry. 2009;80:934-935.
4. Klein CJ, Lennon VA, Aston PA, et al. Insights From LGI1 and CASPR2 potassium channel complex autoantibody subtyping. JAMA Neurol. 2013;70:229-234.

5. Hong MK, Kong J, Namdarian B, et al. Paraneoplastic syndromes in prostate cancer. Nat Rev Urol. 2010;7:681-692.

6. Kobak S. Chronic monoarthritis and foot-drop as a paraneoplastic syndrome in prostate cancer. Rheumatol Int. 2013;33:223-225.

\section{Publish your work in this journal}

Research and Reports in Urology is an international, peer-reviewed, open access journal publishing original research, reports, editorials, reviews and commentaries on all aspects of adult and pediatric urology in the clinic and laboratory including the following topics: Pathology, pathophysiology of urological disease; Investigation and treatment of

\section{Dovepress}

urological disease; Pharmacology of drugs used for the treatment of urological disease. The manuscript management system is completely online and includes a very quick and fair peer-review system, which is all easy to use. Visit http://www.dovepress.com/testimonials.php to read real quotes from published authors.

Submit your manuscript here: http://www.dovepress.com/research-and-reports-in-urology-journal 